\title{
CARACTERÍSTICAS DE LAS EMPRESAS DE BASE TECNOLÓGICA DE ORIGEN UNIVERSITARIO EN MÉXICO
}

\section{CHARACTERISTICS OF TECHNOLOGY-BASED ENTERPRISES OF UNIVERSITY ORIGIN IN MEXICO}

García Martínez, Julio C.* Ramírez Erazo, Abril A.** Cruz Delgado, Daniela.***

\section{RESUMEN}

Las empresas de base tecnológica se han convertido en un tema relevante para los gobiernos y las universidades que buscan impulsar el desarrollo a través del emprendimiento y la innovación. Sin embargo, aún quedan algunas dudas sobre las mejores condiciones para impulsar este tipo de empresas, por ello, el objetivo de esta investigación fue caracterizar a las empresas de base tecnológica de origen universitario (EBT-U) en México enfocándose en algunas variables calificadas como críticas para su emergencia. El alcance de la investigación es descriptivo con una muestra que pertenece a incubadoras de las principales universidades del país y de diversos estados de la república. Principales hallazgos: el 45\% de las EBT-U fueron formadas por emprendedores de nivel licenciatura, el $89 \%$ contaba con experiencia laboral, el 54\% de los emprendedores estudió en el área de ingeniería, 68\% dijo desconocer la política de propiedad intelectual de la universidad, el $52 \%$ cuenta con propiedad intelectual y sólo el $23 \%$ recibió financiamiento adicional al propio, de amigos y familiares.

Palabras clave: Empresa de base tecnológica, emprendedor, políticas universitarias, financiamiento, caracterización.

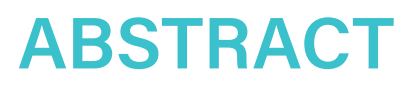

Technology-based companies have become a relevant issue for governments and universities that seek to promote development through entrepreneurship and innovation. However, there are still some doubts about the best conditions to promote this type of companies, therefore, the objective of this research was to characterize technology-based companies of university origin (EBT-U) in Mexico focusing on some variables rated as critics for your emergency. The scope of the research is descriptive with a sample that belongs to incubators of the main universities in the country and various states of the republic. Main findings: $45 \%$ of the EBT-U were formed by entrepreneurs at the undergraduate level, $89 \%$ had work experience, $68 \%$ said they do not know the intellectual property policy of the university, $52 \%$ have intellectual property and only $23 \%$ received additional financing diferent from their own, from relatives or friends.

Keywords: Technology-based company, entrepreneur, university policies, financing, characterization.

\section{AGRADECIMIENTOS}

Se agradece el financiamiento proporcionado por el Programa para el Desarrollo Profesional Docente (PRODEP) para la realización del proyecto con folio UPV-PTC-041 del cual se deriva la presente investigación.

\footnotetext{
*Doctor en Investigación en Ciencias Sociales por FLACSO-México, profesor investigador en Universidad Politécnica de Victoria, jgarciam@upv.edu.mx; **Maestra en Administración de Empresas por La Salle Victoria, profesora investigadora en Universidad Politécnica de Victoria, aramireze@upv.edu.mx; ***Doctora en Problemas Económico Agroindustriales por la Universidad Autónoma Chapingo, profesora investigadora en Universidad Politécnica de Victoria, dcruzd@upv.edu.mx
} 


\section{INTRODUCCIÓN}

Las empresas de base tecnológica se han convertido en un tema relevante para los gobiernos y las universidades que buscan impulsar el desarrollo a través del emprendimiento y la innovación (Fuster et al, 2019; OECD, 2019). Sin embargo, aún quedan algunas dudas sobre las mejores condiciones para impulsar este tipo de empresas, por ello, el objetivo de esta investigación fue caracterizar a las empresas de base tecnológica de origen universitario (EBT-U) en México enfocándose en algunas variables calificadas como críticas para su emergencia. La mayor parte de las investigaciones sobre creación de EBT-Us se han enfocado sólo en algunas variables para explicar su emergencia y en uno o dos niveles de análisis dependido del enfoque teórico elegido. Por lo cual, son escasos los estudios que han abordado el problema de manera comprensiva. En este sentido, se realizó una revisión de la literatura para identificar las variables que desde distintas perspectivas influyen en la creación de EBT-Us. Las variables seleccionadas a partir de la revisión de la literatura fueron: a) micronivel: el área de estudio, el grado de estudios y la experiencia previa del emprendedor; b) mesonivel: política de propiedad intelectual universitaria y oficina de transferencia tecnológica; y c) macronivel: acceso a financiamiento.

La pregunta de investigación que guía esta investigación es ¿cuáles son las principales características de las empresas de base tecnológica de origen universitario en México? La información se recopiló a través de un cuestionario que fue enviado por correo electrónico a los directores de las incubadoras de empresas de alta tecnología, reconocidas por el Instituto Nacional del Emprendedor, ubicadas en las ciudades de México, Puebla, Monterrey, Querétaro, San Luis Potosí, Toluca y Aguascalientes. El alcance de la investigación es descriptivo con sujetos que representan al $25 \%$ de las empresas que se encontraban en incubación en las incubadoras seleccionadas pertenecientes a las principales universidades del país de las ciudades antes mencionadas.

Principales hallazgos: El $82 \%$ de los emprendedores son hombres y el $18 \%$ restante mujeres; por grupo de edad los de mayor participación en la creación de empresas son los de 21 a 30 años con un $36.36 \%$; el $45 \%$ de las EBT-U fueron formadas por emprendedores de nivel licenciatura; el 89\% contaba con experiencia laboral; el 54\% de los emprendedores estudió en el área de ingeniería; el 68\% dijo desconocer la política de propiedad intelectual de la universidad, el 52\% cuenta con propiedad intelectual; sólo el $23 \%$ recibió financiamiento adicional al propio, de amigos y familiares y ninguna empresa que recibió recursos de fondos públicos en su formación accedió a otras fuentes distintas de amigos y familiares; y tampoco lo hicieron en la etapa inicial sin importar si se encontraban en las ciudades con mayor concentración de servicios financieros

El artículo está dividido en cinco apartados. En el segundo a apartado se hace una revisión de la literatura; en el tercero, se presenta la metodología seguida para llevar a cabo la investigación; en el cuarto apartado, se presentan el análisis de resultados y por último se ofrecen algunas conclusiones. 
MICRONIVEL: LAS CARACTERÍSTICAS DE LOS EMPRENDEDORES

Cohen y Levintal, (1990) argumentan que la habilidad de una empresa para reconocer el valor de nueva información, asimilarla y aplicarla a fines comerciales (capacidad de absorción) es crítica para realizar innovación. Esta idea está basada en la capacidad cognitiva del individuo y en sus experiencias previas. En el nivel más elemental, este conocimiento previo incluye habilidades básicas, un lenguaje común y el conocimiento de los desarrollos científicos y tecnológicos más recientes de un campo determinado.

La premisa de la noción de capacidad de absorción es que las organizaciones necesitan conocimientos previos relacionados para asimilar el nuevo conocimiento, esto, basados en los estudios sobre el desarrollo de la memoria.

Cohen y Levintal (1990) señalan que dos ideas están implícitas en la capacidad de absorción: el aprendizaje es acumulativo, y el desempeño en el aprendizaje es mayor cuando el objeto de aprendizaje está relacionado a lo previamente conocido. En consecuencia, mayor acumulación de conocimientos y experiencias facilitan el proceso de innovación porque habilitan a los individuos a hacer nuevas asociaciones y vínculos. En este sentido, el grado y área de estudios del emprendedor, así como su experiencia previa puede ser utilizada como indicadores de su capacidad de absorción, es decir de su mayor o menor capacidad para aplicar comercialmente los conocimientos generados en la universidad. En este sentido, podemos encontrar diversos estudios empíricos que aportan evidencias de la relación de estas variables con la creación de EBT-Us.

\section{Grado académico.}

Los estudios que abordan la relación entre el grado académico y la creación de EBT-Us son realmente escasos y heterogéneos en cuanto a enfoques metodológicos por lo cual sus resultados no son totalmente comparables, por ejemplo, algunos se refieren a la intención de emprender entre profesores-investigadores (Fritsch \& Krabel, 2012) mientras que otros se refieren a personas que ya han iniciado una empresa, entre los cuales se incluyen a los académicos (Åstebro, Bazzazian, y Braguinsky, 2012; Colombo y Delmastro, 2002; Kanellos, 2013)
Colombo y Delmastro (2002) en su estudio sobre incubadoras tecnológicas en Italia, encontraron que los emprendedores incubados iniciaban en mayor medida su negocio para introducir una innovación que los emprendedores que no habían sido incubados; y que además contaban en mayor número de casos con estudios de posgrado. Sin embargo, Fritsch y Krabel (2012) al estudiar la intención de iniciar una empresa entre investigadores hallaron que quienes no tienen doctorado encuentran el inicio de su empresa especialmente interesante y los investigadores senior reportaron significativamente niveles más bajos de atracción por el emprendimiento.

Por su parte, Kanellos (2013) en un estudio longitudinal llevado a cabo en Grecia a los fundadores de empresas tecnológicas para determinar las características de los emprendedores basados en conocimiento encontró que el $27 \%$ de estos contaban con grado equivalente a licenciatura, $33 \%$ con grado de maestría y $14 \%$ con grado de doctor y que $86 \%$ del total de los fundadores tienen al menos educación superior.

De acuerdo a lo que plantea el concepto de capacidad de absorción esperaríamos encontrar que las EBT-U con mayor grado de innovación hayan sido formadas por emprendedores con el grado académico más alto. Sin embargo, los estudios empíricos sólo confirman parcialmente esta hipótesis, ya que efectivamente, han encontrado una relación positiva entre los estudios de posgrado y la creación de EBT-Us pero también se ha encontrado que la mayor proporción de EBT-Us han sido creadas por emprendedores con grado de licenciatura o maestría y no por quienes tienen grado de doctor.

Lo anterior ha sido apoyado por Åstebro et al. (2012) quienes encontraron que $6.4 \%$ de los recién graduados habían iniciado una start-up mientras que ese porcentaje bajaba a $1.3 \%$ entre académicos. Para tener una noción más exacta de la probabilidad de iniciar una start-up por un recién graduado o un académico realizaron un análisis de regresión con un modelo probit mediante el que determinaron que la probabilidad de iniciar una empresa es de 2.5 a 3.5 puntos porcentuales más baja para los académicos y la probabilidad disminuye si el profesor es de tiempo completo. 
Por lo anterior, se espera encontrar que la mayor proporción de EBT-Us hayan sido creadas por emprendedores con estudios de nivel licenciatura.

\section{Área de estudio.}

El perfil científico de la universidad ha sido considerado como un factor que influye en la probabilidad de interactuar con la industria, es decir, que la frecuencia y el tipo de interacciones universidad-empresa para la generación de nuevos emprendimientos está influenciada por el tipo de disciplinas que se imparten en la institución o a la que pertenecen los académicos que desarrollan investigación (D’Este y Patel, 2005). La propensión a comercializar los resultados de la investigación varía considerablemente entre los distintos campos científicos, y se ha encontrado que las ciencias naturales e ingenierías son las que más spin-off generan. Por ejemplo, Golub (2003) (citado por Rodeiro Pazos et al. 2012) reportó que la mitad de todas las empresas que emergieron de Columbia University provenían del área biomédica mientras que el resto provino de la electrónica y software. Shane (2002) halló que la mayoría de las empresas emergidas del MIT operaban en la industria de la biomedicina. Similarmente O'Shea, Allen, Chevalier, \& Roche, (2005) destacó que las investigaciones que se realizan en las ciencias de la salud, informática y química son las que más generan EBTs. Por su parte, D’Este y Patel (2005) encontraron que las disciplinas que más participan en la creación de spin-offs fueron las ingenierías eléctrica y electrónica seguidas por las ingenierías en mecánica aeroespacial y en manufacturas. Así mismo, Rodeiro Pazos et al. (2012) encontraron que las universidades con un fuerte enfoque en las ingenierías y en las ciencias de la vida crean más EBTs debido a que los resultados de sus investigaciones tienen mayores oportunidades de comercialización.

Con base en lo anterior, se espera encontrar que la mayoría de las EBT-Us hayan sido creadas por emprendedores de las áreas de ingeniería y ciencias de la vida y dentro de estas se espera que los de mayor participación sean los de electrónica.

Con base en lo anterior, se espera encontrar que la mayoría de las EBT-Us hayan sido creadas por emprendedores de las áreas de ingeniería y ciencias de la vida y dentro de estas se espera que los de mayor participación sean los de electrónica.

\section{Experiencia previa}

Colombo y Delmastro (2002) encontraron que más del $90 \%$ de los fundadores de las nuevas empresas de base tecnológica contaban con experiencia previa. De ese total, el $45 \%$ había trabajado al interior de alguna empresa y de estos, el $71 \%$ había estado empleado en alguna empresa de alta tecnología. Esta condición también fue observada por (Kodama, 2008), quien señala que, en TAMA Japón, las pequeñas empresas desarrolladoras de productos han sido fundadas principalmente por ingenieros que dejaron sus trabajos en las grandes empresas eléctricas y electrónicas afectadas por la crisis del petróleo en los años setentas. En el caso de las spin-off universitarias, estudiadas por Rasmussen y Borch (2010), pudo observarse cómo sus fundadores contaban con experiencia previa en activiades de investigación y/o en la industria, lo que facilitó la comercialización de los conocimientos. Por otro lado, Schleinkofer y Schmude (2013) estudiaron 173 proyectos de EBT-Us en Alemania en los que midieron la influencia de diversos factores sobre la creación de la empresa entre los que se encontraba la experiencia previa (experiencia en proyectos de investigación, en la industria y en emprendimiento) medida como capital humano; sin que se encontrara alguna influencia entre estas variables.

${ }^{1}$ Este porcentaje no incluye a los profesores que han abierto una empresa pero reportaron a la universidad como su principal empleador. 
Aunque los resultados de Schleinkofer y Schmude (2013), sustentados con rigor estadístico, contradicen la suposición de que la experiencia está relacionada positivamente con la creación de EBTUs, estos están acotados a la población estudiada. Por lo cual, se espera encontrar evidencia de la relación entre experiencias previas y la formación de EBT-Us.

\section{MESONIVEL: POLÍTICAS Y ESTRUCTU- RAS DE APOYO UNIVERSITARIAS.}

Los estudios sobre la transferencia de conocimientos han identificado el papel que juegan las políticas y estructuras organizacionales, como facilitadores en la comercialización de los conocimientos, entre las cuales podemos destacar a las políticas de propiedad intelectual y las oficinas de transferencia tecnológica.

\section{Política de propiedad intelectual}

Un factor clave en la comercialización de la investigación universitaria ha sido el cambio en la legislación sobre los derechos de propiedad intelectual. La Bayh-Dole Act (1980) de los Estados Unidos permitió a los investigadores financiados con recursos públicos patentar los resultados de sus investigaciones y licenciarlos a terceras partes. Esta ley instituyó los incentivos para que las universidades se apropiaran de las innovaciones y fomentó el crecimiento del número de patentes registradas por las universidades (CEPAL, 2010).

La introducción de estos cambios en la legislación respondieron a la necesidades de hacer frente a una creciente demanda de investigación tecnológica por parte de los sectores más dinámicos y modernos, como la nanotecnología y la biotecnología, que se encontraban en un estado incipiente y a la creciente participación de la universidades estadounidenses en el patentamiento desde 1963 (CEPAL, 2010; Leydesdorff y Meyer, 2010). Los supuestos efectos de la Bayh-Dole Act, sobre el patentamiento universitario en los Estados Unidos, han llevado a otros gobiernos a introducir legislaciones similares.

Generalmente, los derechos sobre los ingresos generados por el licenciamiento son repartidos entre el inventor y varias partes de la universidad. Utilizando datos de 102 universidades estadounidenses para el periodo 1991-1999 Lach y Schankerman (2003) encontraron que las universidades que otorgan una mayor participación a los académicos en los ingresos obtenidos por el licenciamiento generan más invenciones y más ingresos por licencia. La política de propiedad intelectual es un factor que ha facilitado la comercialización de los conocimientos, no sólo a las universidades estadounidenses sino también en otros lugares como en Canadá (Bramwell y Wolfe, 2008; Landry, Amara, y Ouimet, 2007), en Japón (Kodama, 2008) y Europa (Debackere, 2010; Fini, $\mathrm{Fu}$, Mathisen, Rasmussen, \& Wright, 2017). Así mismo, Muscio, Quaglione, y Ramaciotti (2016) demostraron que, en Italia los incentivos económicos, como la participación de los investigadores en las regalías obtenidas por la comercialización de la propiedad intelectual, tenía efectos positivos en la creación de spin-offs.

Esto implica que el diseño de las políticas de propiedad intelectual en las universidades tiene efectos reales sobre la productividad académica en la transferencia conducida por incentivos económicos. Por lo tanto, se espera encontrar que las universidades que tengan establecidas políticas de propiedad intelectual que otorgan participación a los investigadores en la obtención de regalías generen más EBT-Us y que a mayor participación mayor número de EBT-Us creadas.

\footnotetext{
${ }^{2}$ La comercialización de la investigación universitaria entendida como las actividades relacionadas con el patentamiento, el licenciamiento de invenciones y la creación de empresas a diferencia de la vinculación académica o 'academic engagement' que involucra otras formas de transferencia como investigación contratada y conjunta, consulto-
}

rías y otras (Perkmann et al., 2013) 
Oficinas de transferencia tecnológica.

Aunado al diseño de políticas de propiedad intelectual, y los incentivos para que los académicos estrechen su relación con la industria, se encuentran las estructuras organizacionales como las oficinas de transferencia tecnológica (OTT), las oficinas de enlace u otros centros cuya finalidad explícita es la comercialización de la propiedad intelectual generada por la institución. Estas estructuras proveen de recursos y consultoría a los académicos y estudiantes emprendedores, y se encargan de proteger y administrar la propiedad intelectual (Fuster et al., 2019; Rasmussen y Borch, 2010).

Por ejemplo, algunas universidades, como Geogia Tech, no esperan a que los académicos tomen el primer paso de revelar sus invenciones a las oficinas de transferencia sino que monitorean activamente la investigación académica y alientan a los investigadores a realizar los descubrimientos (Rothaermel y Thursby, 2005). Otro ejemplo es la OTT de la Universidad Católica de Lovaina, en Bélgica, que ha estimulado la explotación de la investigación universitaria a través de una rica mezcla de mecanismos ${ }^{5}$ para estimular el emprendimiento entre sus distintas divisiones, convirtiéndose en un caso de éxito en la Unión Europea (Debackere, 2010). Algunos estudios como el de Fini et al. (2017) llevado a cabo en Italia, Noruega y Reino Unido aportan evidencia del papel que juegan la OTTs en la creación de EBT-Us. Así mismo, Berbegal-Mirabent, Ribeiro-Soriano, y Sánchez García (2015) encontraron, en España, que el apoyo brindado por las OTT mediante personal especializado tiene efectos positivos en la creación de EBT-Us. En el caso de México, la Red de Oficinas de Transferencia Tecnológica reportó a través de una encuesta que realiza entre sus miembros que las OTT's habían contribuido a formar 65 empresas de base tecnológica en el 2015 y 168 en 2017 (red OTT, 2015; 2018), lo cual representa una participación creciente en la creación de EBT's.
Por lo anterior, se espera encontrar evidencia que confirme la influencia positiva de las OTT sobre la creación de EBT-Us.

${ }^{3}$ Lach y Schankerman (2003) señalan que la actividad de licenciamiento ha crecido dramáticamente en las pasadas dos décadas en los Estados Unidos. Las patentes concedidas a los inventores universitarios creció de 500 en 1982 a más de 3100 en 1998, y el número de licencias creció de 1278 a 4362 durante la última década.

${ }^{4}$ En las universidades estudiadas por Lach y Shankerman la participación de los académicos en las regalías oscilaba de $25 \%$ hasta $65 \%$.

${ }^{5}$ La OTT de Lovaina ha desarrollado cuatro actividades principales: negociación y desarrollo de investigación contratada y conjunta, protección y licenciamiento de la propiedad intelectual, desarrollo de planes de negocios para la creación de spin-offs y la generación de una sola plataforma de innovación abierta para la universidad.

${ }^{6}$ Aunque del total de OTT's sólo el 34\% están relacionadas con instituciones de educación superior públicas y privadas, $35 \%$ son de iniciativa privada y el resto es de gobierno y otros. 
Aunque las EBT-Us son impulsoras del cambio tecnológico y de la creación de empleos, se enfrentan con dificultades para acceder al financiamiento convencional que otorga el sector bancario y el mercado de valores; incluso estas dificultades pueden ser mayores a las que enfrentan las nuevas y pequeñas empresas de los sectores tradicionales que ofrecen productos o servicios ya probados en el mercado.

Por ello, la existencia de instituciones que ofrecen capital semilla y de riesgo a las empresas spin off son esenciales para su sobrevivencia, ya que por su tamaño, poca experiencia y por ofrecer productos aun no probados en el mercado resultan ser inversiones más riesgosas y que difícilmente pueden cubrir los requisitos y las condiciones que establece la banca comercial (Carlsson 2002; Colombo y Delmastro, 2002; Fuentes y Ampudia, 2009).

Bozkaya y Van Pottelsberghe (2008) afirman que las características asociadas con las nuevas empresas de base tecnológica tales como la opacidad de su información; el alto riesgo e incertidumbre, el largo plazo requerido para desarrollar el potencial económico; la falta inicial de activos tangibles; la falta de registros contables; y las asimetrías de información generan problemas de financiamiento particulares para estas empresas.

En un estudio realizado por Harvard Business School encontraron que más del $60 \%$ de las incubadoras en el mundo nunca pudieron conseguir financiamiento para sus empresas incubadas (Aernoudt, 2004). Por su parte Bozkaya y Van Pottelsberghe (2008) después de estudiar 107 pequeñas empresas de base tecnológica en Bélgica, encontraron que su principal fuente de financiamiento en la etapa de formación eran los recursos propios seguidos de préstamos de amigos y familiares. En México, Corona (1997); estudió cien empresas innovadoras en el que describe sus principales características, se encontró que el principal obstáculo para las micro y pequeñas empresas fue el acceso al financiamiento. Como muestran los estudios antes citados, en la etapa de formación prevalece como principal o única fuente de financiamiento el capital propio, de amigos $y$ familiares.

En este mismo sentido Avdeitchikova (2009) señala que, en Suecia, las nuevas empresas de base tecnológica se enfrentan a dificultades de financiamiento debido a que el mercado de capital de riesgo informal se encuentra altamente concentrado en las áreas metropolitanas y ciudades universitarias. Así mismo, Murray (1998) señala que las concentraciones espaciales existentes en la oferta de capital de riesgo perjudicaron la formación de nuevas empresas innovadoras en las regiones de la Unión menos desarrolladas económicamente. Debido a esto la Unión Europea creó el European Seed Capital Fund Pilot Scheme (1988-95) como una respuesta a las disparidades en la oferta de capital de riesgo para las nuevas empresas de base tecnológica. De manera que la existencia de inversionistas ángel y fondos de capital en el entorno cercano es un factor que facilita el acceso a financiamiento para estas empresas. Por lo cual, esperamos encontrar más EBT-Us financiadas con capital de riesgo en las ciudades con mayor presencia de este tipo de actores.

Por su parte, la Comisión Europea ha señalado que uno de los principales obstáculos para la creación de Spin-outs académicos en Europa es la falta de recursos financieros apropiados. Y que la falta de financiamiento para las primeras etapas de formación y crecimiento de las empresas se debe a los bajos rendimientos que a menudo se obtienen de tales inversiones. Por lo cual, esperar tasas de retorno equiparables a las del mercado es un criterio inapropiado para juzgar la rentabilidad generada por las inversiones realizadas en las primeras etapas del ciclo de vida de la empresa. Para salvar estos obstáculos, se ha planteado la provisión de 
subsidios gubernamentales, la participación de fondos de capital de riesgo dispuestos a invertir en las primeras etapas de la empresa y la participación de inversionistas ángel que aporten capital semilla (European Commission, 2006).

El efecto de los subsidios públicos sobre las restricciones financieras-de las pequeñas NTBF también ha sido documentado por otros estudios, por ejemplo, Meuleman y De Maeseneire (2012) señalan que la obtención de préstamos del gobierno para I+D provee una señal positiva sobre la EBT y genera un efecto positivo para obtener créditos de largo plazo. De manera semejante Colombo, Croce, y Guerini (2013) encontraron que la recepción de subvenciones públicas por parte de pequeñas empresas de base tecnológica da como resultado un aumento de la tasa de inversión. Estos resultados apoyan la opinión de que los subsidios públicos pueden ayudar a estas empresas a superar los obstáculos para acceder al financiamiento externo.

Esquemas de financiamiento que proporcionen los recursos en la etapa pre-comercial y de crecimiento inicial con capital semilla son esenciales en las etapas tempranas de la empresa; y para la etapa de expansión (posterior al proceso de incubación) se ha señalado como indispensable la existencia de un mercado bien desarrollado de capital de riesgo, como el NASDAQ y el NASDAQ Europa, ya que estos proveen el capital accionario que requieren las empresas de nuevas tecnologías (Djokovic y Souitaris, 2008). En este sentido, se espera encontrar, en general, una baja participación del capital de riesgo en la estructura de financiamiento de las EBT-Us pero una estructura de financiamiento más diversificada en aquellos que han recibido subsidios del gobierno. 


\section{METODOLOGÍA}

El alcance de la investigación es exploratorio en el sentido del uso que se puede dar a los resultados y descriptivo en el sentido de la técnica de análisis utilizada ya que no se cuenta con una muestra representativa de la población y sólo se utiliza la estadística descriptiva para mostrar algunos hallazgos que tendrán que ser constatados con estudios posteriores. La información se recopiló a través de un cuestionario que fue enviado por correo electrónico a los directores de las incubadoras de empresas de alta tecnología reconocidas por el Instituto Nacional del Emprendedor ubicadas en las ciudades de México, Puebla, Monterrey, Querétaro, San Luis Potosí, Toluca y Aguascalientes; las primeras cuatro ciudades se encuentran entre las primeras 7 que más solicitudes de propiedad intelectual por parte de mexicanos realizaron. Los Directores de las incubadoras a su vez lo reenviaron a los emprendedores que en ese momento tenían alguna relación con cada incubadora. Los cuestionarios fueron aplicados durante los meses de abril, mayo y junio de 2017.

El cuestionario aplicado incluye 34 ítems, de los cuales 3 se refieren a datos demográficos del emprendedor, 5 para experiencia, 8 para conocer el grado de innovación y características del producto o proceso, 4 para indagar si la idea de negocio fue producto de la transferencia tecnológica, 2 relativos a la OTT, 1 respecto a la política de propiedad intelectual de la universidad, 5 respecto al financiamiento, y 6 respecto a las características de la empresa. La mayoría de los ítems fueron preguntas cerradas.

Se consiguieron 22 respuestas, lo que representa un $25.6 \%$ de la población total de EBT-Us que se encontraban en incubación .

Tabla 1. Variables que empíricamente se ha encontrado que influyen en la creación de EBT-Us de acuerdo a nivel de análisis y corriente.

\begin{tabular}{|l|l|l|}
\hline Niveles & Corrientes & Variables \\
\hline \multirow{2}{*}{ Macro } & \multirow{2}{*}{ Influencia ambiental externa } & Mercado financiero o acceso al financiamiento \\
\hline Meso & $\begin{array}{l}\text { Determinantes institucionales y } \\
\text { organizacionales }\end{array}$ & Oficina de Transferencia Tecnológica \\
\cline { 3 - 3 } & & Políticas de propiedad intelectual \\
\hline Micro & $\begin{array}{l}\text { Atributos o características } \\
\text { individuales }\end{array}$ & Nivel de estudios \\
\cline { 3 - 3 } & & Grado de estudios \\
\cline { 3 - 3 } & & Experiencia \\
\hline
\end{tabular}

Fuente: elaboración propia con base en Djokovik y Souitaris (2008) y O'Shea et al. (2008) 


\section{RESULTADOS}

El $82 \%$ de los emprendedores son hombres y el 18 restante mujeres, lo que refleja una gran brecha por cubrir en el emprendimiento por género, al menos en lo referente a EBTs ya que en un estudio realizado sobre la microempresa en México reveló que las mujeres participan como propietarias en por lo menos el $50 \%$ de los micronegocios (INEGI, 2012; Pinzón, Guzmán, y López; 2017; Posada, Aguilar, y Peña; 2018) ; por grupo de edad los de mayor participación en la creación de empresas son los de 21 a 30 años con un $36.36 \%$ y en segundo lugar los de 31 a 40 años de edad con un $27.27 \%$. El $45 \%$ cuenta con nivel licenciatura, $18 \%$ maestría y $27 \%$ con doctorado y el resto cuenta con educación de nivel media superior. Casi el $50 \%$ de la EBT-Us han sido fundadas por emprendedores con nivel licenciatura en línea con lo encontrado por Åstebro et al. (2012).

El $71.3 \%$ de las empresas han sido fundadas por dos o más emprendedores lo que resulta positivo ya que se pueden compartir riesgos y complementar recursos (tabla 2). El $77.3 \%$ cuenta con hasta 5 trabajadores por lo cual la gran mayoría nace y funciona como microempresa durante sus primeras etapas de vida (tabla 3). El $45 \%$ de las EBT pertenecen al sector industrial, muy por arriba de la participación de este sector en la actividad económica general, esto resalta la importancia de este tipo de empresas para el desarrollo de la industria en México (gráfica 1).

Tabla 2. Número de emprendedores que fundaron la empresa

\begin{tabular}{|c|c|c|c|c|}
\hline & 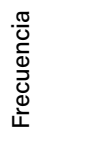 & $\begin{array}{l}\frac{0}{\pi} \\
\frac{0}{0} \\
\frac{0}{0} \\
\frac{0}{0} \\
0\end{array}$ & 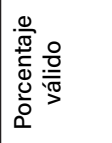 & 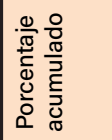 \\
\hline 1 & 5 & 22.7 & 22.7 & 22.7 \\
\hline 2 & 7 & 31.8 & 31.8 & 54.5 \\
\hline 3 & 4 & 18.2 & 18.2 & 72.7 \\
\hline 4 & 5 & 22.7 & 22.7 & 95.5 \\
\hline 5 & 1 & 4.5 & 4.5 & 100.0 \\
\hline Total & 22 & 100.0 & 100.0 & \\
\hline
\end{tabular}

Fuente: elaboración propia
Tabla 3. Número de personas que laboran en la empresa

\begin{tabular}{lrrrr}
\hline & Frecuencia & Porcentaje & $\begin{array}{c}\text { Porcentaje } \\
\text { válido }\end{array}$ & $\begin{array}{r}\text { Porcentaje } \\
\text { acumulado }\end{array}$ \\
\hline 1 & 1 & 4.5 & 4.5 & 4.5 \\
2 & 2 & 9.1 & 9.1 & 13.6 \\
3 & 6 & 27.3 & 27.3 & 40.9 \\
4 & 5 & 22.7 & 22.7 & 63.6 \\
5 & 3 & 13.6 & 13.6 & 77.3 \\
6 & 1 & 4.5 & 4.5 & 81.8 \\
7 & 1 & 4.5 & 4.5 & 86.4 \\
8 & 1 & 4.5 & 4.5 & 90.9 \\
10 & 1 & 4.5 & 4.5 & 95.5 \\
14 & 1 & 4.5 & 4.5 & 100.0 \\
Total & 22 & 100.0 & 100.0 & \\
\hline
\end{tabular}

Fuente: elaboración propia.

Gráfica 1. Sector de actividad de las EBT-U

Agropecuaria 4\%

Servicio $50 \%$

Industria 46\%

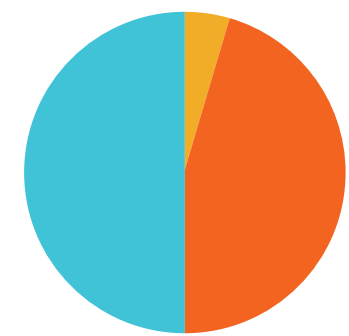

En cuanto a la suposición de que la mayor parte de las EBT-Us hayan sido formadas por emprendedores con grado de licenciatura se cumplió ya que estos fundaron el $45 \%$ del total, como se señaló líneas arriba; mientras que los productos con mayor innovación (midiendo el grado de innovación por la propiedad intelectual y el alcance geográfico) fueron generados por quienes tenían grado de doctor, como se muestra en la tabla 4, y sólo 2 emprendedores tienen registrada su PI en México y el extranjero de los cuales uno tiene grado de doctor y otro de maestría (tabla 5).

Tabla 4. Grado de estudios alcanzado y de propiedad intelectual (PI)

\begin{tabular}{l|c|c|c|}
\hline \multirow{2}{*}{} & \multicolumn{2}{|c|}{$\begin{array}{c}\text { Producto con PI otorgada o } \\
\text { en trámite }\end{array}$} & Total \\
\cline { 2 - 4 } & No & Sí & \\
\hline Doctorado & 1 & 5 & 6 \\
Maestría & 0 & 4 & 4 \\
$\begin{array}{l}\text { Licenciatura / } \\
\text { Ingeniería }\end{array}$ & 8 & 2 & 10 \\
$\begin{array}{l}\text { Media superior / } \\
\text { Técnico Superior }\end{array}$ & 1 & 1 & 2 \\
Total & 10 & 12 & 22 \\
\hline
\end{tabular}

Fuente: elaboración propia

${ }^{7}$ El sector manufactura sólo participa con el $16 \%$ en el total de micronegocios del país (INEGI; 2012) 
Tabla 5. Grado de estudios alcanzado y países donde se encuentra registrada la propiedad intelectual que protege la innovación.

\begin{tabular}{|c|c|c|c|c|c|}
\hline & \multirow[t]{2}{*}{ Sin $\mathrm{PI}$} & \multicolumn{3}{|c|}{$\begin{array}{l}\text { ¿En qué países se } \\
\text { encuentra registrada } \\
\text { la propiedad intelec- } \\
\text { tual que protege su } \\
\text { innovación? }\end{array}$} & \multirow[t]{2}{*}{ Total } \\
\hline & & México & $\begin{array}{l}\text { Otro } \\
\text { país. }\end{array}$ & & \\
\hline \multirow{4}{*}{$\begin{array}{l}\text { 2. Indi- } \\
\text { que su } \\
\text { máximo } \\
\text { grado de } \\
\text { estudios } \\
\text { alcanzado }\end{array}$} & Doctorado & 1 & 4 & 1 & 6 \\
\hline & $\begin{array}{l}\text { Licenciatura / } \\
\text { Ingeniería }\end{array}$ & 8 & 2 & 0 & 10 \\
\hline & Maestría & 0 & 3 & 1 & 4 \\
\hline & $\begin{array}{l}\text { Media superior } \\
\text { / Técnico Sup. }\end{array}$ & 1 & 1 & 0 & 2 \\
\hline Total & & 10 & 10 & 2 & 22 \\
\hline
\end{tabular}

Fuente: elaboración propia

El 54\% de los emprendedores estudiaron en el área de ingeniería, le siguen los de ciencias de la vida con $18 \%$ y el resto en otras áreas, en línea con estudios anteriores (D'Este y Patel, 2005; O'Shea et al., 2005; Rodeiro Pazos et al., 2012; Shane, 2002). Sin embargo, el área de electrónica no fue la subárea con mayor participación como se esperaba.

Respecto a la experiencia previa, se encontró que el $95 \%$ contaba con experiencia antes de iniciar su negocio, el $72 \%$ del total tenía experiencia en investigación y el $40.9 \%$ en industria en concordancia con los estudios de Colombo y Delmastro (2002), Kodama (2008) y Rasmussen y Borch (2010) (tabla 6). Esto significa, evidentemente, que una buena parte de los emprendedores tiene experiencia laboral tanto en la industria como en investigación, destacando esta última. Sorprendentemente sólo el $36.4 \%$ de los emprendedores dijeron que su producto o servicio se había generado a partir de la investigación desarrollada en la universidad, lo cual indica una moderada transferencia tecnológica a través de empresas o la negación al origen de la investigación por el posible conflicto de interés universidad-investigador.

Tabla 6. Participación porcentual de los encuestados en las características seleccionadas.

\begin{tabular}{l|c|c|c} 
& & No & Sí \\
\hline $\begin{array}{l}\text { Experiencia laboral anterior al inicio } \\
\text { de su empresa }\end{array}$ & 4.5 & 95.5 \\
\hline $\begin{array}{l}\text { En alguno de los puestos que ha } \\
\text { desempeñado realizó actividades } \\
\text { de investigación y desarrollo }\end{array}$ & 4.5 & 22.7 & 72.7 \\
\hline $\begin{array}{l}\text { Alguna de las empresas donde la- } \\
\text { boró pertenece al sector industrial }\end{array}$ & 4.5 & 54.5 & 40.9
\end{tabular}

Fuente: elaboración propia
Para encontrar evidencias de la influencia que tiene la política de propiedad intelectual al interior de la universidad sobre la generación de innovaciones se preguntó a los emprendedores si al momento de iniciar su empresa conocían el porcentaje de participación en las regalías que otorga la universidad a los investigadores que participaron en la investigación. El resultado fue que el $68 \%$ de los emprendedores dijo desconocer ese dato y el $18 \%$ mencionó $0 \%$, así que podríamos afirmar que a la mayoría de los emprendedores parecen no importarle los incentivos económicos en contraste con lo planteado por Bramwell y Wolfe (2008), Debackere (2010), Fini et al. (2017), Landry et al. (2007), Kodama (2008), y Muscio et al. (2016), sin embargo, hay que tomar en cuenta que solo el $34 \%$ de los emprendedores dijo que las innovaciones surgieron de la investigación universitaria, pero de ese total sólo el $37 \%$ señaló conocer el porcentaje de participación.

Por otro lado, el $63 \%$ de los emprendedores señaló que la universidad de donde provienen cuenta con OTT (tabla 7), pero sólo $36 \%$ ha utilizado sus servicios en concordancia con el porcentaje de innovaciones derivadas de la investigación universitaria. Esto se puede interpretar como señala Rodeiro Pazos et al. (2012) factores que en algunos ambientes inciden de forma positiva en la creación de empresas, no lo hacen en otros. El anterior es el caso del efecto de las OTT's en las universidades con menos conocimiento o entendimiento de los negocios como el caso de España, en donde su presencia no incide de manera positiva en la creación de spin-offs mientras que, en otros ambientes, donde la universidad cuenta con más conocimiento de negocios como en Estados Unidos, sí se encuentra una relación positiva, de ahí la importancia de estudiar cada ambiente en lo particular. La evidencia parece indicar que para las empresas derivadas de la investigación universitaria sí es importante la función de las OTT's mas no para las que sólo han utilizado el servicio de incubación.

Tabla 7. La universidad donde realizó sus estudios ¿cuenta con oficina de transferencia tecnológica (OTT)?

\begin{tabular}{ccccc}
\hline & Frecuencia & Porcentaje & $\begin{array}{c}\text { Porcentaje } \\
\text { válido }\end{array}$ & $\begin{array}{c}\text { Porcentaje } \\
\text { acumulado }\end{array}$ \\
\hline No & 8 & 36.4 & 36.4 & 36.4 \\
Sí & 14 & 63.6 & 63.6 & 100.0 \\
Total & 22 & 100.0 & 100.0 & \\
\hline
\end{tabular}

Fuente: elaboración propia 
En cuanto al financiamiento, se corrobora el supuesto de que el capital de riesgo distinto del propio, familiares y amigos se encuentra ausente de la estructura de financiamiento de las EBT-U en la etapa de formación en línea con hallazgos anteriores (Aernoudt, 2004; Bozkaya y Van Pottelsberghe, 2008; Corona, 1997; OECD, 1999). Sólo el $13 \%$ dijo haber recibido financiamiento adicional al propio, de familiares y amigos en la etapa de formación (sin incluir los fondos públicos) e inicial. El $27 \%$ recibió financiamiento del Consejo Nacional de Ciencia y Tecnología de sus diversos programas y $4.5 \%$ de la Secretaría de Economía. Contradictoriamente a lo que se esperaba, ninguna empresa que recibió recursos de fondos públicos en su formación accedió a otras fuentes distintas de amigos y familiares; y tampoco lo hicieron en la etapa inicial sin importar si se encontraban en las ciudades con mayor concentración de servicios financieros. Las fuentes de financiamiento convencionales como los bancos y los no convencionales como inversionistas ángel, el capital amigable (crowfunding), y los fondos de inversión siguen estando ausentes en la etapa temprana de las empresas (en la tabla 8 se muestran algunos resultados).

Tabla 8. Fuentes de financiamiento antes de iniciar operaciones.

\begin{tabular}{|c|c|c|}
\hline & Frecuencia & Porcentaje \\
\hline Propio, Familiares y Amigos & 18 & 82 \\
\hline $\begin{array}{l}\text { Propio, Amigos, Fondo de In- } \\
\text { versión Privado }\end{array}$ & 1 & 4.5 \\
\hline $\begin{array}{l}\text { Propio, Familiares, Amigos, } \\
\text { Banca privada }\end{array}$ & 1 & 4.5 \\
\hline $\begin{array}{l}\text { Propio, Familiares, Amigos, } \\
\text { Crowdfunding (financia- } \\
\text { miento masivo: Kickstarter } \\
\text { u otra plataforma), Banca } \\
\text { privada }\end{array}$ & 1 & 4.5 \\
\hline $\begin{array}{l}\text { Propio, Familiares, Amigos, } \\
\text { SECRETARÍA DE ECONO- } \\
\text { MÍA }\end{array}$ & 1 & 4.5 \\
\hline Total & 22 & 100.0 \\
\hline
\end{tabular}

Fuente: elaboración propia 
La creación de empresas de base tecnológica de origen universitario o university spin-off como se les conoce en el idioma anglosajón, es un fenómeno social y como tal, se ve afectado por múltiples factores de los cuales, en el presente estudio, sólo se han abordado algunos de los más citados en la literatura internacional y de contextos diferentes. Por ello, los diversos estudios que abordan la creación de EBT-U sólo han coincidido parcialmente, ya sea porque las variables se han medido de manera diferente o porque la calidad de las variables no es la misma. Los hallazgos aquí presentados sugieren que los recién egresados son un recurso importante para impulsar la creación de este tipo de empresas y debe ser tomado en cuenta por los hacedores de políticas. Se debe tomar en cuenta que la menor proporción serán innovaciones más radicales a través de los recursos humanos altamente capacitados y de investigaciones de calidad generadas en la universidad.

Es evidente que el área de ingeniería tiene mayores oportunidades de generar innovaciones, pero a diferencia de la experiencia internacional, en los casos aquí estudiados, la mayor parte no se concentra en la electrónica, sino que existe una participación muy diversa de ingenierías. No se puede afirmar que sin experiencia en la industria o en investigación no hay EBT-Us, pero ha resultado muy ilustrativo que más del $90 \%$ de los emprendedores contaba con alguno de estos tipos de experiencia previa.

En relación a las políticas y estructuras implementadas por la universidad para alentar y facilitar la creación de EBTs, los resultados arrojados convergen parcialmente con lo planteado por la literatura, ya que sólo el $34 \%$ de las empresas se han derivado de la investigación universitaria, en concordancia con el $36 \%$ de los emprendedores que han utilizado servicios de una OTT. Por otro lado, la política de propiedad intelectual parece no estar teniendo algún efecto relevante en este fenómeno. Aunque este dato debería tomarse con precaución ya que en México la implementación de políticas de propiedad intelectual al interior de las universidades es muy reciente, incluso aún no están bien definidas, ya que fue hasta finales del año 2015 que se realizaron reformas a la Ley de Ciencia y Tecnología y a la Ley de Federal de Responsabilidades Administrativas de los Servidores Públicos que permiten la explotación comercial de la propiedad intelectual generada y la creación de empresas derivadas de la investigación en instituciones de educación y centros de investigación públicos.

Por último, no se encontró evidencia positiva entre financiamiento público y acceso a otras fuentes y se confirma que el acceso a fuentes de financiamiento en condiciones favorables sigue siendo un problema para las pequeñas empresas, sobre todo en las etapas tempranas y para las EBT-Us. Por ello, se requiere de un verdadero esfuerzo por la política pública y la sociedad civil para impulsar la emergencia de capital emprendedor tanto público como privado, pero sobre todo de este último, en la figura de inversionistas ángel, crowfunding y fondos de inversión dispuestos a asumir mayores niveles de riesgo que impulsen la creación de nuevas empresas basadas en el avance de la ciencia y la tecnología. 


\section{REFERENCIAS}

Aernoudt, R. (2004). "Incubators : Tool for Entrepreneurship ?" en Small Business Economics, Sep 2004(23, 2), 127-135.

Åstebro, T., Bazzazian, N., y Braguinsky, S. (2012). "Startups by recent university graduates and their faculty: Implications for university entrepreneurship policy" en Research Policy, 41(4), 663677. https://doi.org/10.1016/j.respol.2012.01.004

Avdeitchikova, S. (2009). "False expectations: Reconsidering the role of informal venture capital in closing the regional equity gap" en Entrepreneurship and Regional Development, 21(2), 99-130. https://doi.org/10.1080/08985620802025962

Berbegal-Mirabent, J., Ribeiro-Soriano, D. E., y Sánchez García, J. L. (2015). "Can a magic recipe foster university spin-off creation?" en Journal of Business Research, 68(11), 2272-2278. https:// doi.org/10.1016/j.jbusres.2015.06.010

Bercovitz, J., y Feldman, M. (2003). "Technology transfer and the academic department: who participates and why" en DRUID Summer Conference, 12-14. https://doi.org/10.1017/ CBO9780511618390.021

Bramwell, A., y Wolfe, D. A. (2008). "Universities and regional economic development: The entrepreneurial University of Waterloo" en Research Policy, 37(8), 1175-1187. https://doi.org/10.1016/j. respol.2008.04.016

Caiazza, R. (2014). "Factors affecting spin-off creation: Macro, meso and micro level analysis" en Journal of Enterprising Communities, 8(2), 103-110. https://doi.org/10.1108/JEC-12-20120061

Carlsson, B. (2002). "Institutions, Entrepreneurship,and Growth: Biomedicine and Polymers in Sweden and Ohio" en Small Business Economics, 105-121.

CEPAL. (2010). Espacios Iberoamericanos. Vínculos entre universidades y empresas para el desarrollo tecnologico, CEPAL, Santiago de Chile.

Cohen, W. M., y Levintal, D. A. (1990). "Absorptive
Capacity: A New Perspective on Learning and Innovation" en Administrative Science Quarterly, 35(1), 128-152. https://doi.org/10.2307/2393553

Colombo, M. G., y Delmastro, M. (2002). "How effective are technology incubators?" en Research Policy, 31(7), 1103-1122. https://doi. org/10.1016/S0048-7333(01)00178-0

Colombo, M. G., Croce, A., y Guerini, M. (2013). "The effect of public subsidies on firms' investment-cash flow sensitivity: Transient or persistent?" en Research Policy, 42(9), 1605-1623. https://doi.org/10.1016/j.respol.2013.07.003

Corona Treviño, L. (1997) Cien empresas innovadoras en México,UNAM; M.A. Porrúa, México.

D'Este, P., y Patel, P. (2005). "SPRU Science and Technology Policy Research University of Sussex Falmer" en Science And Technology, (July), 1-26.

Debackere, K. (2010). "The rise of the academic technology transfer organization" en Review of Business and Economics, LV(April-June), 175189.

Djokovic, D., y Souitaris, V. (2008). "Spinouts from academic institutions: A literature review with suggestions for further research" en Journal of Technology Transfer, 33(3), 225-247. https:// doi.org/10.1007/s10961-006-9000-4

European Commission. (2006). Financing Technology Transfer. Ssrn, (November 2006). https:// doi.org/10.2139/ssrn.1846344

Fini, R., Fu, K., Mathisen, M. T., Rasmussen, $E_{\text {., }} \mathrm{y}$ Wright, M. (2017). "Institutional determinants of university spin-off quantity and quality: a longitudinal, multilevel, cross-country study" en Small Business Economics, 48(2), 361-391. https://doi. org/10.1007/s11187-016-9779-9

Fritsch, M., y Krabel, S. (2012). "Ready to leave the ivory tower?: Academic scientists' appeal to work in the private sector" en The Journal of Technology Transfer, 37(3), 271-296. https://doi. org/10.1007/s10961-010-9174-7

Fuentes, Claudia de, y Lourdes Ampudia. (2009) "Los sistemas regionales de innovación de Querétaro y Ciudad Juárez" en Gabriela Dutrénit, Sistemas Regionales de Innovación:un espacio para 
el desarrollo de las pymes. El caso de la industria de maquinados industriales, ( 81-107),UAM-TEXTUAL, México.

Fuster, E.; Padilla-Meléndez, A.; Lockett, N.; DelÁguila-Obra, A. R. (2019). "The emerging role of university spin-off companies in developing regional entrepreneurial university ecosystems: The case of Andalusia" en Technological Forecasting and Social Change, 141, 219-231. https:// doi.org/10.1016/j.techfore.2018.10.020

INEGI (2012). Encuesta nacional de micronegocios 2012. Recuperado de http://www.beta.inegi. org.mx/programas/enamin/2012/

Kanellos, N. S. (2013). "Exploring the Characteristics of Knowledge-based Entrepreneurs in Greece" en Procedia - Social and Behavioral Sciences, 73, 337-344. https://doi.org/10.1016/j. sbspro.2013.02.060

Kodama, T. (2008). "The role of intermediation and absorptive capacity in facilitating university-industry linkages-An empirical study of TAMA in Japan" en Research Policy, 37(8), 1224-1240. https://doi.org/10.1016/j.respol.2008.04.014

Lach, S., y Schankerman, M. (2003). "INCENTIVES AND INVENTION IN UNIVERSITIES" en NBER WORKING PAPER SERIES, 9727. Recuperado de https://www.nber.org/papers/w9727. pdf

Landry, R., Amara, N., y Ouimet, M. (2007). "Determinants of knowledge transfer: Evidence from Canadian university researchers in natural sciences and engineering" en Journal of Technology Transfer, 32(6), 561-592. https://doi.org/10.1007/ s10961-006-0017-5

Leydesdorff, L., y Meyer, M. (2010). "The decline of university patenting and the end of the Bayh $\mathrm{C}^{-}$ Dole effect" en Scientometrics, 83(2), 355-362. http://dx.doi.org/10.1007/s11192-009-0001-6

Markuerkiaga, Caiazza, R., Igartua, J., y Nekane;, E. (2016). "Factors fostering students' spin-off firm formation: An empirical comparative study of universities from North and South" en Journal of Management Development 35(6). http:// dx.doi.org/10.1108/JMD-03-2016-0034
Mayer, C. (2002). "Financing the new economy: Financial institutions and corporate governance" en Information Economics and Policy, 14(2), 311-326. https://doi.org/10.1016/S01676245(01)00072-5

Meuleman, M., y De Maeseneire, W. (2012). "Do R\&D subsidies affect SMEs' access to external financing?" Research Policy, 41(3), 580-591. https://doi.org/10.1016/j.respol.2012.01.001

Murray, G. C. (1998). "A policy response to regional disparities in the supply of risk capital to new technology-based firms in the European Union: the European Seed Capital Fund Scheme" en Regional Studies, 32(5), 405-419. https://doi. org/10.1080/00343409850116817

Muscio, A., Quaglione, D., y Ramaciotti, L. (2016). "The effects of university rules on spinoff creation: The case of academia in Italy" en Research Policy, 45(7). https://doi.org/10.1016/j.respol.2016.04.011

OECD (2019), University-Industry Collaboration : New Evidence and Policy Options, OECD Publishing, Paris.

https://doi.org/10.1787/e9c1e648-en

O'Shea, R. P., Allen, T. J., Chevalier, A., y Roche, F. (2005). "Entrepreneurial orientation, technology transfer and spinoff performance of U.S. universities" en Research Policy, 34(7), 994-1009. https://doi.org/10.1016/j.respol.2005.05.011

O'Shea, R. P., Chugh, H., y Allen, T. J. (2008). "Determinants and consequences of university spinoff activity: A conceptual framework" en Journal of Technology Transfer, 33(6), 653-666. https:// doi.org/10.1007/s10961-007-9060-0

Perkmann, M., Tartari, V., Mckelvey, M., Autio, E., Broström, A., Este, P. D., $\square$ Sobrero, M. (2013). Academic engagement and commercialisation : A review of the literature on university - industry relations. Research Policy, 42(2), 423-442. https://doi.org/10.1016/j.respol.2012.09.007

Pinzón Castro, S. Y., Maldonado Guzmán, G., \& López Torres, G. C. (2017). "Caracterización de la muestra de las microempresas en México" en G. Maldonado Guzmán, J. F. Ojeda Hidalgo, L. J. Uc Heredia, L. E. Valdéz Juárez, \& M. Medina Elizondo, La microempresa en México: un diagnóstico 
de su situación actual (págs. 37-58), Universidad Autónoma de Aguascalientes. México

Posada Velázquez, R., Aguilar Rascón, Ó. C., \& Peña Ahumada, N. B. (2016). Análisis sistémico de la micro y pequeña empresa en México, Pearson, México.

Rasmussen, E., y Borch, O. J. (2010). "University capabilities in facilitating entrepreneurship: $A$ longitudinal study of spin-off ventures at mid-range universities" en Research Policy, 39(5), 602612. https://doi.org/10.1016/j.respol.2010.02.002

Rodeiro Pazos, D., Fernández López, S., Otero González, L., y Rodríguez Sandiás, A. (2012). "A resource-based view of university spin-off activity: New evidence from the Spanish case" en Revista Europea de Dirección y Economía de La Empresa, 21(3), 255-265. https://doi.org/10.1016/j.redee.2012.05.006

Rothaermel, F. T., y Thursby, M. (2005). "University-incubator firm knowledge flows: Assessing their impact on incubator firm performance" en Research Policy, 34(3), 305-320. https://doi.or$\mathrm{g} / 10.1016 / \mathrm{j}$.respol.2004.11.006

Schleinkofer, M., y Schmude, J. (2013). "Determining factors in founding university spinoffs" en International Journal of Entrepreneurship and Small Business, 18(4), 400. https://doi. org/10.1504/IJESB.2013.053484

Shane, S. (2002). "Selling University Technology: Patterns from MIT" en Management

Science, 48(1), 122-137. https://doi.org/10.1287/ mnsc.48.1.122.14281 